Bibliotecas. Vol 40, № 1, enero - junio, 2022. EISSN: 1659-3286

URL: http://www.revistas.una.ac.cr/index.php/bibliotecas/index

DOI: http://dx.doi.org/10.15359/rb.40-1.2

Licencia: Creative Commons (BY-NC-SA) 4.0 Internacional

\title{
Habilidades informativas y tecnológicas de la población estudiantil de Bibliotecología de la UNAM ${ }^{1}$
}

Information and Technological Skills of UNAM's Library Students

\author{
Selene Violeta Castillo Rojas \\ Colegio de Bibliotecología, UNAM, México \\ ORCID $\underline{0000-0002-8333-7383}$
}

Carlos Omar Sánchez Xicotencatl

Facultad de Psicología, UNAM, México

ORCID $\underline{0000-0002-7767-0040}$

Alberto Castro Thompson

Instituto de Investigaciones Bibliográficas, UNAM

ORCID $\underline{0000-0001-7298-2742}$

Lina Escalona Ríos

Facultad de Filosofía y Letras, UNAM, México

ORCID $\underline{0000-0002-5109-4612}$

Recibido: 30 de noviembre de 2020

Aceptado: 08 de setiembre de 2021

Publicado: 24 de enero de 2022

\begin{abstract}
Resumen
Las tecnologías de la información y comunicación (TIC) se han convertido en un elemento clave para mejorar el proceso educativo, y los recursos documentales en formato digital son el eje de dicho proceso, por lo que el alumnado debe adquirir y/o desarrollar una serie de habilidades tecnológicas e informativas que le permitan obtener información de calidad, con validez académica y pertinencia para sus actividades académicas. El presente trabajo hace referencia a un diagnóstico sobre las habilidades que poseen los estudiantes de Bibliotecología y Estudios de la Información de la Universidad Nacional Autónoma de México, que admitan establecer estrategias para optimizar del desarrollo de dichas habilidades.
\end{abstract}

Palabras clave: habilidades informativas, habilidades tecnológicas, Bibliotecología. 


\begin{abstract}
Information and communications technology (ICT) have become an important key to improve the educative process and documentary resources in digital format are the axis of this process, so students must acquire and / or develop a series technological and information skills that allow you to obtain quality information, with academic validity and relevance for your academic activities. This work refers to a diagnosis of the skills that students of Library Science and Information Studies of the National Autonomous University of Mexico possess, which allow establishing strategies to optimize the development of these skills.
\end{abstract}

Keywords: information skills, technology skills, Library Science.

\title{
I. Introducción
}

El uso de Tecnologías de la Información y Comunicación (TIC) en la educación es una práctica cotidiana. En distintos países existe una gran diversidad de programas y proyectos que impulsan su uso para los procesos de enseñanza y de aprendizaje, especialmente en cuanto a los recursos bibliográficos y/o documentales, los cuales se han convertido en un requerimiento básico para que la población estudiantil y el personal docente consulten información con validez académica, pertinente y relevante para sus actividades académicas. Lo anterior ha llevado a toda una corriente, en el ámbito bibliotecológico, sobre la alfabetización informacional, alfabetización de la información en España y habilidades informativas en América Latina, lo que implica su definición teórica y alcance hasta su parte práctica al establecer los cursos de Desarrollo de Habilidades Informativas (DHI) para una comunidad determinada.

Más allá de la discusión teórica sobre los conceptos anteriores, estos se han asociado indiscutiblemente con las TIC, por lo que la población usuaria o estudiantil también debe tener una serie de habilidades tecnológicas que le permitan avanzar hacia las habilidades informativas. Entonces, el presente trabajo parte de la importancia que han tenido las TIC para incorporarse a los planes de desarrollo en México y, con base en su importancia, llegar a una conceptualización de las habilidades tecnológicas e informativas con la finalidad de analizar las habilidades que presenta el estudiantado de Bibliotecología de la UNAM.

Para lograr el objetivo, durante septiembre y octubre del 2019, se eligió una muestra no probabilística de 152 estudiantes. Se aplicó un cuestionario conformado por 217 reactivos en los salones de clase con previa autorización del profesorado y la coordinación de la licenciatura. 


\section{Habilidades tecnológicas desde los planes de desarrollo en México}

En México, los planes de educación planteados, desde hace varios años, enfatizan la importancia de implementar mejores estrategias de aprendizaje, la ampliación de la cobertura de los servicios educativos, asignar mayores recursos económicos y la implementación de herramientas tecnológicas. Desde finales de la década de 1960, se incorporaron oficialmente el uso de tecnologías en el ámbito educativo con la implementación de las telesecundarias como alternativa para que los habitantes de zonas rurales tuvieran acceso a la educación. En 1994 inicia una red de educación por televisión (EDUSAT) con una cobertura en todo México (Durán, Rivero, Alfaro y Gayosso, 2018). En el programa de educación 1995 - 2000, se enfatiza el fomento y apoyo al uso de herramientas tecnológicas, en uno de sus apartados se menciona: Independientemente de la naturaleza y objetivos de los programas académicos, se alentará la formación integral de los estudiantes con una visión humanista y responsable frente a las necesidades y oportunidades del desarrollo de México. En este marco, se dará énfasis al desarrollo de la creatividad, dominio del español, pensamientos lógico y matemático. Además, se apoyarán acciones que atiendan la habilitación de los estudiantes en informática y lenguas extranjeras, entre otras áreas. (Secretaría de Educación Pública, s/fa, p.3)

Dentro del mismo programa, se indica la importancia de los medios tecnológicos para que más estudiantes accedan a la educación, y su uso especialmente desde los primeros años, con la finalidad de incrementar el interés por la ciencia y la tecnología. También, se pretendía utilizar la tecnología por parte de los docentes: "Los medios electrónicos se utilizarán con toda intensidad para contribuir al logro de los objetivos del sistema educativo nacional.... estos instrumentos constituirán un valioso complemento a la labor docente, tanto en modalidades escolarizadas, como en mixtas y no escolarizadas" (Secretaría de Educación Pública, 1995, p. 140).

Por otro lado, en el sexenio del 2000 al 2006, se retoma la importancia de incluir nuevas tecnologías en la educación; por ejemplo, se inició con el programa e-México que tenía un fin educativo y en el 2003 se inició el programa de Enciclomedia, el cual, para los responsables del 
ámbito educativo, representaba un elemento muy importante en la mejora de la educación en México. La Enciclomedia fue anunciada como:

[...] una herramienta didáctica desarrollada por científicos e investigadores mexicanos, que relaciona los contenidos de los libros de texto gratuito con el programa oficial de estudios y diversos recursos tecnológicos como audio y video, a través de enlaces de hipermedia que conducen al estudiante y maestro a un ambiente atractivo, colaborativo y organizado por temas y conceptos que sirvieron de referencia a recursos pedagógicos relacionados con el currículo de educación básica. (Secretaría de Educación Pública, $\mathrm{s} / \mathrm{fb}, \mathrm{p} .11)$

Si bien el programa fue una estrategia educativa con alta expectativa, careció de indicadores que permitieran conocer el impacto real en el nivel educativo de los jóvenes que fueron beneficiados con la Enciclomedia (Azamar, 2016). En el programa de educación del 2007-2012, además de dar importancia a la responsabilidad social del alumnado, se buscó la incorporación de las tecnologías en las aulas para apoyar los procesos de aprendizaje de la población estudiantil con la finalidad de mejorar la calidad educativa e incrementar las competencias relacionadas al uso de las TIC, uno de los objetivos principales del programa de educación fue: "Impulsar el desarrollo y utilización de tecnologías de la información y la comunicación en el sistema educativo para apoyar el aprendizaje de los estudiantes, ampliar sus competencias para la vida y favorecer su inserción en la sociedad del conocimiento" (Secretaria de Educación Pública, 2007, p.11).

Asimismo, vale la pena analizar el estudio realizado por las Naciones Unidas y Comisión Económica para América Latina y el Caribe (Hinostroza y Labbé, 2011) que incluyó a 17 países de Latinoamérica, donde se reportó que México es uno de los países que presenta altos indicadores acerca de la incorporación de las TIC en educación. El país se destaca por una política publicada e institucionalizada, además tiene el mayor número de objetivos a cumplir y presenta el mayor número de acciones para lograrlo. Igualmente, se reporta que el $63 \%$ de las escuelas cuentan con al menos una computadora y $67 \%$ de las escuelas, con acceso a internet, y un $68 \%$ de las escuelas tiene recursos digitales, pero solo un $33 \%$ de los docentes habían sido capacitados para el uso de las TIC; no se reportó el porcentaje de estudiantes capacitado 
para el uso de TIC. Así, México aparece como un país con altos indicadores de definición e implementación de la política TIC.

Durante el periodo 2000 al 2012 en México surgieron programas específicos con énfasis en el desarrollo de tecnologías e infraestructura, como "e-México" (Secretaría de Comunicaciones y Transportes, 2001) y "Agenda Digital.mx" (Secretaría de Comunicaciones y Transportes, 2012). Ambos programas presentan marcadas similitudes en cuanto a promover la transformación de la sociedad mexicana, considerando conectividad, ampliación de ancho de banda, impulsar el desarrollo de servicios y contenidos digitales y, por supuesto, promover el uso de las TIC en la educación, la economía y, en general, en todos los sectores del gobierno. Sin dejar de considerar, líneas específicas relacionadas con inclusión social y brecha digital.

La política educativa del periodo 2012 al 2018 también señaló la importancia del uso de las tecnologías en la educación, para ello en 2013 la Dirección General de Tecnologías de la Información y Comunicación (DGTIC) inició actividades con el objetivo de:

Proveer y administrar las tecnologías de información y los sistemas de telecomunicaciones necesarios para que las unidades administrativas desarrollen sus funciones, y para brindar seguridad y confianza en el manejo de la información, mediante el fortalecimiento de un gobierno digital y abierto que induzca una mayor participación de los ciudadanos. (Gobierno de México, s/f)

Dentro de las múltiples funciones de la DGTIC, se encuentran coordinar diferentes sistemas informáticos, proveer los medios a las instituciones y promover el uso de tecnología, entre otras.

En México se cuenta con una agenda digital que marca la pauta del uso de TIC ahora denominadas TICCAD (Tecnologías de la Información, Comunicación, Conocimiento y Aprendizaje Digital); actualmente, la educación digital se encuentra dentro de la ley general de educación, donde se establece que "toda persona tiene derecho a gozar de los beneficios del desarrollo de la ciencia y la innovación tecnológica", además que "el Estado garantizará el derecho de acceso a las tecnologías de la información y comunicación, así como a los servicios 
de radiodifusión y telecomunicaciones, incluido el de banda ancha e internet" (Secretaria de Educación Pública, s/fc, pp.16-17).

Los resultados de las actuales políticas de educación son aún incipientes, sin embargo, es importante señalar que el uso de las TIC se ha convertido en un eje fundamental del modelo educativo. Al respecto, Hernández (2017) menciona que "la tecnología se ha convertido en el motor central de oportunidades, que permiten la oportunidad de innovar en educación, donde los resultados obtenidos, producto de este impacto científico deberán buscar la solución a problemas sociales-educativos útiles para el desarrollo" (p. 329).

En general, se observa que desde hace varios años el uso de las TIC cada vez se presenta como un tema importante y recurrente en el ámbito educativo, sin embargo, las diferencias existentes de las TIC en acceso, uso, conocimientos y habilidades, así como en infraestructura institucional e intereses personales, establecen brechas significativas entre sectores de la población, dando lugar a diversas investigaciones del uso de TIC en la educación, donde predominan los estudios en el nivel superior (Olivares, Angulo, Torres y Madrid, 2016), las cuales consideran las habilidades que se requieren para su uso ya que son consideradas transversales dentro del currículo.

Por consiguiente, es necesario incrementar la investigación en niveles básico y medio superior para conocer los efectos del uso de las TIC desde edades tempranas, aplicando estudios longitudinales que podrían dar evidencia de la evolución de las habilidades y conocimientos necesarios requeridos por la comunidad estudiantil para utilizar, de manera eficaz, la tecnología en la educación.

\section{Habilidades tecnológicas e informativas}

La población estudiantil de nivel universitario de la actualidad pertenece a una generación en la que ya nacieron con las tecnologías a su alrededor. El celular lo manejan desde pequeños, las computadoras, tablet, laptop, etcétera, con acceso a internet, son recursos que usan para comunicarse o para jugar sin mayor problema. Se puede afirmar, de acuerdo con Prensky (2001) que son "nativos digitales" y tienen una serie de habilidades tecnológicas; aunque 
también existen los "inmigrantes digitales" quienes se adaptan al entorno tecnológico, aunque tienen reticencias de su pasado conocimiento de la cultura impresa, como el imprimir los correos.

Algunos investigadores han enfatizado la importancia y la robustez de los recursos y las competencias. En este sentido y de acuerdo con Prahalad y Hamel (1990), citados por Castellanos y otros (2009), es necesario clarificar los constructos de "recursos, capacidades, competencias, competencias nucleares (core competences) y competencias distintivas" ( $p$. 134), estableciendo la diferencia entre ellos para determinar de cuál se está hablando en este estudio. De esta forma, los recursos son objetos o sujetos, tangibles o intangibles, por ejemplo: recursos humanos o intelectuales.

Por otra parte, la capacidad es una característica innata para desempeñar cualquier actividad; mientras que la competencia se adquiere con la práctica continua de una actividad y al conjunto de competencias importantes para el logro de un objetivo se le llaman competencias nucleares o core competences (Coombs, 1996, citado por Castellanos y otros, 2009).

Dentro de las competencias nucleares se encuentran las competencias o habilidades tecnológicas definidas como la capacidad de identificar y manejar los conceptos y aplicaciones vinculadas a las tecnologías de la información, no solo del hardware, sino también del software y los principales medios de comunicación a través de estos medios. De acuerdo con la Matriz de habilidades TIC para el aprendizaje (Ministerio de Educación, 2013), aplicada a un grupo de estudiantes en Santiago de Chile, las habilidades tecnológicas se caracterizan por:

- Tener conocimientos TIC: se refiere a la capacidad de manejar y entender conceptos TIC utilizados para nombrar las partes y funciones de los computadores y las redes...

- Operar las TIC: considera la capacidad de usar las TIC de forma segura, de resolver problemas técnicos básicos y de administrar información y archivos.

- Usar las TIC: se refiere a la habilidad de dominar software, hardware y programas de uso extendido en la sociedad, particularmente aquellos que facilitan el aprendizaje individual y con otros. (p. 20) 
Entre las habilidades tecnológicas sobre las que se preguntó al estudiantado, se encuentran:

- Aplicación de las herramientas.

- Uso de office.

- Uso de presentaciones.

- Manejo de navegadores y búsqueda en Internet.

- Uso de redes sociales.

Por otra parte, las habilidades informativas $(\mathrm{HI})$ han sido dirigidas en su mayoría por las bibliotecas, las cuales han desarrollado modelos y estándares sobre estas. En la literatura se pueden encontrar diversas definiciones como la que ofrece el Chartered Institute of Library and Information Professionals (2004) quien señala que la alfabetización informacional (ALFIN) hace referencia a saber cuándo y por qué se requiere información, así como el lugar en donde encontrarla, la forma de evaluación, de uso y de comunicación.

Webber (2006) considera que las habilidades informativas se refieren a la adopción de un comportamiento apropiado para obtener recursos que satisfagan las necesidades de información. Mientras que Freire y Mora (2009) proponen tres aspectos: habilidades tecnológicas, competencias comunicativas y sociales, análisis crítico sobre las implicaciones éticas y políticas de la tecnología y sus usos. González (2010) señala que las habilidades pueden localizarse en diferentes niveles: a) sensibilización, b) conocimiento de las prácticas, c) dominio de las herramientas y d) dominio metodológico.

Sobre el concepto de habilidades informativas, diferentes autores y asociaciones han asumido diferentes definiciones. La Asociación Americana de Bibliotecas Escolares (AASL), precursora en este campo, y la Asociación para las Comunicaciones y Tecnologías Educativas, señalan que "Habilidad informativa o habilidad informacional es (la habilidad para encontrar y usar información) la piedra angular en el aprendizaje permanente" (Byerly/Brodie, 1999, p. 11).

Bajo el componente de habilidades informativas, AASL afirma que "los estudiantes con habilidades informativas acceden a la información efectiva y eficientemente, evalúan la 
información de manera crítica y competente y la utilizan de manera creativa y precisa" (Ibíd.). Las personas usuarias "deberían tener tanto estrategias para recabar información como las habilidades de pensamiento crítico para seleccionar, descartar, sintetizar y presentar información en formas nuevas para resolver problemas de la vida real” (Ibíd., p. 11). Así, se concuerda con que definir a las habilidades informativas "se extiende más allá de las habilidades de uso en la biblioteca, y más allá de estrategias y habilidades particulares a la habilidad de utilizar información compleja de diferentes fuentes, para desarrollar significado o resolver problemas" (Kuhlthau, citado en Lau, 2007, p. 11).

Autores como Owusu-Ansah (2003) han dejado claro que se ha llevado a cabo un gran trabajo para consensuar el término de habilidades informativas, pero que aún existen diferencias entre las definiciones propuestas. La más comúnmente utilizada y citada es la adoptada por la Asociación Bibliotecaria Americana, ALA en 1988 (American Library Association): "para ser un individuo con habilidades informativas (alfabetizado informacionalmente), una persona debe ser capaz de reconocer cuando necesita información y tener la habilidad para localizar, evaluar y utilizar efectivamente la información necesaria. Los individuos con habilidades informativas son aquellos que han aprendido a aprender" (Citado en Lau, 2007, p. 12). Y saben cómo aprender porque saben cómo está organizado el conocimiento, cómo encontrar información y cómo usar la información de tal modo que otros puedan aprender de ellos (Byerly/Brodie, 1999). Cualesquiera que sean las consideraciones semánticas que asumamos para los términos "habilidades informativas/informacionales". La definición de ALA es lo suficientemente amplia como para abarcar todo el espectro de las competencias en información o alfabetización informacional: desde la sabiduría tradicional de los Inuit hasta los motores de búsqueda de alta tecnología, y probablemente esta conceptualización sea aplicable durante varias décadas (Campbell, 2004).

Por otra parte, en el Proyecto DeSeCo, de la OCDE (2002) se menciona que "cada competencia reposa sobre una serie de habilidades prácticas y cognitivas interrelacionadas: conocimientos, motivación, valores, actitudes, emociones y otros elementos sociales y de comportamiento que pueden ser movilizados conjuntamente para actuar de manera eficaz" (p. 8) 
Para efectos de este trabajo se vinculan las competencias y las habilidades, por lo que se considera a las habilidades informativas como el conjunto de características que posee la persona para buscar, localizar y obtener información digital, pertinente, relevante con validez académica para los fines que el usuario requiera.

\section{Análisis de resultados}

La muestra de estudiantes seleccionada está conformada por 152 estudiantes de la carrera de Bibliotecología y Estudios de la Información de la UNAM, el $36.5 \%$ son hombres y $63.5 \%$ mujeres; distribuidos por semestre de la siguiente forma: segundo semestre el $23 \%$, cuarto semestre el $27.6 \%$, sexto semestre el $22.4 \%$ y octavo semestre el $27 \%$. Con un rango de edad de 18 a 58 años ( $m=22.8$, de $=5.3$ ) de los turnos: matutino el $50 \%$, vespertino con $39.5 \%$ y mixto $10.5 \%$. Cabe estacar que la encuesta se llevó a cabo antes de iniciar la contingencia sanitaria en México provocada por el COVID-19, por lo que los datos obedecen a un contexto escolarizado y presencial.

Los participantes reportaron que las bibliotecas digitales que más utilizan corresponden al Sistema Bibliotecario de la UNAM (SIBIUNAM) con $52.4 \%$, seguidas de las que pertenecen a instituciones tanto privadas como públicas (34.8\%); también se reportó que el $12.8 \%$ de las personas participantes no consultan bibliotecas digitales. Esto se puede deber a que la comunidad académica de Bibliotecología tiene acceso a una colección especializada en la Biblioteca Samuel Ramos de la Facultad de Filosofía y Letras y en la Biblioteca Central de la UNAM, además de tener acceso a una excelente colección impresa en la Biblioteca del Instituto de Investigaciones Bibliotecológicas y de la Información.

También, se puede observar que es elevado el porcentaje de estudiantes que acceden a bibliotecas digitales, ya que la facilidad que se ofrece para acceder a los recursos las hace de fácil consulta. Sobre el uso de bases de datos especializadas, destacan Scielo (30.3\%), LISA (15.9\%), INFOBILA (14.8\%), IIBI (4.7\%), Redalyc (4.7\%), EBSCO (4.2\%) y otras $(25.4 \%)$. Además, para facilitar el acceso a la información, el 18.9\% de los participantes siempre marca un sitio o página web, $36.5 \%$ lo hace de forma frecuente, $25.7 \%$ poco frecuente, $13.5 \%$ de manera ocasional y el 5.4\% nunca. Lo anterior indica la claridad que tiene el estudiantado sobre 
las bases de datos especializadas en su disciplina, excepto el $4.7 \%$ que responde el IIBI refiriéndose a un instituto y no a una base de datos.

En el tema de la comunicación y colaboración, más del $90 \%$ de la población estudiantil de los diferentes semestres ocupa el correo electrónico para enviar trabajos a los profesores. Lo anterior es importante porque siendo una licenciatura de modalidad presencial, es de llamar la atención el hecho de enviar los trabajos al profesorado por este medio cuando, tradicionalmente, se hacía de forma impresa.

Uno de los temas de mayor interés fue el uso de redes sociales, donde Facebook se posicionó en primer lugar con un $77 \%$, seguida de WhatsApp con 33\%, Instagram en un $41 \%$ y Twitter con $22 \%$. Los principales usos que les dan son entretenimiento en $78.2 \%$, estar en contacto con los amigos en $71.7 \%$, ver qué publican los demás en $39.4 \%$ y asuntos laborales en $32.2 \%$. Las principales ventajas de usarlas fueron enterarse más fácil y rápido de las cosas que suceden en el mundo con $69.7 \%$, comunicarse con las personas que se encuentran lejos en $52 \%$ y que ayudan mucho en el trabajo o negocio con solo $20.4 \%$.

Hay que hacer notar la función de las redes sociales ya que, como se ve en estos datos, su uso fundamental es para comunicación social, no para uso académico necesariamente. En relación con la evaluación de las redes sociales, el $83.1 \%$ las considera una muy buena herramienta, el $14.7 \%$ que presentan más riesgos que aspectos positivos y $2.2 \%$ que no traen nada bueno. En cuanto a su uso como medio de comunicación, el 37.5\% opina que es igual que hacerlo en persona, por lo que el $28.3 \%$ prefiere comunicarse en persona. El $17.8 \%$ mencionó que es más fácil que en persona y el $15.8 \%$ reporta que es fácil comunicarse a través de las redes pero que no les cuesta trabajo hacerlo en persona.

Cuando se cuestionó si el uso de redes sociales había cambiado la forma de estudiar, el 45.4\% mencionó que el cambio fue parcial, el $13.8 \%$ completo, un $23 \%$ no cambió y el $17 \%$ no lo había considerado. Esto concuerda con la opinión de la población estudiantil sobre el uso, ya que como se indicó es para comunicación social. 
En el tema de antivirus, el $51 \%$ reportó utilizar uno gratuito, $42.2 \%$ de tipo comercial y $6.8 \%$ copia ilegal. Además, el 34.5\% verifica mensualmente que el equipo esté libre de virus informático, mientras que el $26.4 \%$ lo hace semestralmente, $18.9 \%$ semanal y el $20.3 \%$ del estudiantado no lo verifica. En este rubro es fundamental que todos los estudiantes que participaron en la encuesta han considerado el programa de antivirus como parte de su seguridad informática más allá del tipo de adquisición realizada.

Al utilizar unidades de almacenamiento extraíbles, la población estudiantil reportó que verifican la probabilidad de virus en un $61.6 \%$, mientras el resto menciona no hacerlo (38.4\%). Ante esa situación, se preguntó con qué frecuencia pierden información por causa de virus, destacando que en un $45.6 \%$ no le sucede, $35.6 \%$ en ocasiones, $16.1 \%$ poco frecuente y solo el $2.7 \%$ reportó que era frecuente.

Se cuestionó acerca de la información que registran en las cuentas de correo y redes sociales, encontrando que el $52.7 \%$ mencionó que los datos que proporcionan son una mezcla de verídicos y ficticios, el $40 \%$ mencionó registrar únicamente verídicos y el $6 \%$ especificó solo ficticios. La configuración de parámetros de seguridad la establecen de manera inmediata (59.9\%), o bien, se deja pasar un tiempo (18.4\%), aunque existe un $21.7 \%$ que no sabe qué debe hacer al respecto. El $82.9 \%$ no ha sido víctima de usurpación de identidad digital mientras que el $17.1 \%$ reporta algún tipo de experiencia.

Con el propósito de conocer cuáles equipos de cómputo poseen los estudiantes, se encontró que un $52 \%$ tiene tablet, un $79.6 \%$ equipo portátil, el $55.9 \%$ equipo de escritorio, un $35.5 \%$ escáner, el 50.7\% impresora y un 32.9\% multifuncional. Se puede observar que tres terceras partes de la muestra poseen equipo portátil lo que implica la facilidad para la realización de actividades académicas.

En cuanto a los dispositivos de almacenamiento más frecuentes, se tiene que en primer lugar se encuentran los dispositivos USB (53\%), seguido del celular (18.4\%), nube (14\%), disco externo (12.3\%) y en último lugar el CD-ROM (11.1\%). Al preguntar sobre los servicios de la nube que se utilizan desde el celular, se reportó que principalmente es la computadora (36.9\%) 
seguido de Drive (31.5\%). Los equipos con los que se sincroniza el celular en casa son la computadora $(38.9 \%)$ y la tablet $(9.3 \%)$. Si se tuviera que reestablecer el celular, el $53 \%$ mencionó que lo llevaría al técnico, mientras que un 47\% intenta repararlo por sí mismo.

Además, que el $70.8 \%$ no tiene instalado un antivirus en ese equipo, solo el $29.2 \%$ si lo tiene. En cuanto a la frecuencia con la que se eliminan archivos temporales de internet en el equipo de cómputo, se reportó que el $48 \%$ lo hace semestralmente, el $7.9 \%$ anual, el $14.9 \%$ desconoce el procedimiento a seguir y el $29.6 \%$ no lo hace nunca. Las aplicaciones fundamentales que el celular debe tener son redes sociales (38.3\%) y Google (8\%), mientras que aquellas utilizadas con mayor frecuencia en apoyo a las actividades académicas son Drive (13.2\%) y Google (11.5\%). Al realizar una comparación entre hombres y mujeres, se observaron algunas diferencias (Cuadro 1).

\section{Cuadro 1}

Aplicaciones frecuentes en actividades académicas

\begin{tabular}{|c|c|c|c|c|c|c|c|c|c|c|c|c|c|c|c|c|c|c|}
\hline \multirow[t]{2}{*}{ Semestre } & \multicolumn{2}{|c|}{ Correo } & \multicolumn{2}{|c|}{ Drive } & \multicolumn{2}{|c|}{ Edmodo } & \multicolumn{2}{|c|}{$\begin{array}{c}\text { Whats- } \\
\text { App }\end{array}$} & \multicolumn{2}{|c|}{ Office } & \multicolumn{2}{|c|}{ Google } & \multicolumn{2}{|c|}{$\begin{array}{c}\text { Facebo } \\
\text { ok }\end{array}$} & \multicolumn{2}{|c|}{ Adobe } & \multicolumn{2}{|c|}{$\begin{array}{l}\text { You- } \\
\text { Tube }\end{array}$} \\
\hline & $\mathrm{H}^{*}$ & $\mathrm{M}$ & $\mathrm{H}$ & $\mathrm{M}$ & $\pi$ & $\mathrm{M}$ & $\mathrm{H}$ & $M$ & 11 & $\mathrm{IVI}$ & $\mathrm{H}$ & $M$ & $\mathrm{H}$ & $\mathrm{M}$ & $\mathrm{H}$ & $M$ & $\mathrm{H}$ & $M$ \\
\hline & & 0 & & & & & & 1 & & & & 13 & 17 & 13 & & & 16 & \\
\hline Cual & $L^{2}$ & T & & 2 & & & & I & & & & 27 & 17 & 10 & 25 & & & 10 \\
\hline Sext & 0 & 0 & 2 & 2 & & 14 & & 0 & $2 v$ & 20 & DI & 29 & 0 & 0 & & & & 0 \\
\hline Jctavc & 46 & 18 & 46 & 6 & c & 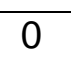 & 1 & 23 & 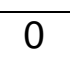 & 14 & 16 & 18 & 11 & 18 & & 14 & 0 & 14 \\
\hline
\end{tabular}

* Para este cuadro, la "H" se refiere a Hombres y la "M" a Mujeres.

Fuente: elaboración propia de las personas autoras, 2020.

Una vez que se describió el panorama del estudiantado con relación al uso de TIC, se reporta el nivel de las habilidades evaluadas por género y semestre. Al comparar otros aspectos relevantes en el uso de TIC, como los motores de búsqueda, las acciones que realizan después de entrar a un sitio web, las estrategias utilizadas para buscar información en Internet y los criterios utilizados para evaluar información, nuevamente se observaron diferencias por sexo y semestre (Cuadros 2, 3, 4 y 5). 


\section{Cuadro 2}

Motores de búsqueda utilizados por la población estudiantil

\begin{tabular}{|l|c|c|c|c|}
\hline \multirow{2}{*}{ Semestre } & \multicolumn{2}{|c|}{ Google } & \multicolumn{2}{c|}{ Facebook } \\
\cline { 2 - 5 } & Hombres & Mujeres & Hombres & Mujeres \\
\hline Segundo & 83.3 & 78.3 & 8.3 & 13 \\
\hline Cuarto & 75 & 90 & 16.7 & 0 \\
\hline Sexto & 76.9 & 100 & 7.7 & 0 \\
\hline Octavo & 78.9 & 86.4 & 5.3 & 0 \\
\hline
\end{tabular}

Fuente: elaboración propia de las personas autoras, 2020.

Cuadro 3

Acciones realizadas después de entrar en un sitio web

\begin{tabular}{|l|c|c|c|c|c|c|}
\hline \multirow{2}{*}{ Semestre } & \multicolumn{2}{|l|}{$\begin{array}{l}\text { Lees y regresas a } \\
\text { seguir buscando }\end{array}$} & \multicolumn{2}{c|}{ Lees y eliges los enlaces } & \multicolumn{2}{l|}{$\begin{array}{l}\text { Lees y navegas hasta } \\
\text { lograr el objetivo }\end{array}$} \\
\cline { 2 - 7 } & Hombres & Mujeres & Hombres & Mujeres & Hombres & Mujeres \\
\hline Segundo & 16.7 & 56.5 & 41.7 & 26.1 & 41.7 & 17.4 \\
\hline Cuarto & 25 & 45.5 & 50 & 22.2 & 25 & 32.3 \\
\hline Sexto & 27.3 & 28.6 & 31.8 & 28.6 & 40.9 & 42.9 \\
\hline Octavo & 57.9 & 27.3 & 10.5 & 31.8 & 31.5 & 40.9 \\
\hline
\end{tabular}

Fuente: elaboración propia de las personas autoras, 2020.

\section{Cuadro 4}

Estrategias para la búsqueda en internet

\begin{tabular}{|l|c|c|c|c|c|c|}
\hline \multirow{2}{*}{ Semestre } & \multicolumn{2}{|c|}{ Palabras clave } & \multicolumn{2}{c|}{ Frases } & Frases entre comillas \\
\cline { 2 - 7 } & Hombres & Mujeres & Hombres & Mujeres & Hombres & Mujeres \\
\hline Segundo & 75 & 82.6 & 25 & 13 & 0 & 4.3 \\
\hline Cuarto & 91.7 & 78.9 & 4.1 & 15.5 & 4.2 & 5.6 \\
\hline Sexto & 61.5 & 61.9 & 23.1 & 33.3 & 15.4 & 4.8 \\
\hline Octavo & 78.4 & 4.5 & 21.1 & 72.7 & 0 & 22.7 \\
\hline
\end{tabular}

Fuente: elaboración propia de las personas autoras, 2020. 


\section{Cuadro 5}

Criterios imprescindibles al calificar como confiable a la información

\begin{tabular}{|c|c|c|c|c|c|c|c|c|c|c|c|c|c|c|c|c|}
\hline \multirow[t]{2}{*}{ Semestre } & \multicolumn{2}{|c|}{$\begin{array}{l}\text { Sitio web } \\
\text { confiable }\end{array}$} & \multicolumn{2}{|c|}{$\begin{array}{c}\text { Autor } \\
\text { reconocido }\end{array}$} & \multicolumn{2}{|c|}{ Instituciones } & \multicolumn{2}{|c|}{ Referencias } & \multicolumn{2}{|c|}{ Año } & \multicolumn{2}{|c|}{ Ortografía } & \multicolumn{2}{|c|}{$\begin{array}{l}\text { Fecha de } \\
\text { publicación }\end{array}$} & \multicolumn{2}{|c|}{ Citas } \\
\hline & $\mathrm{H}^{*}$ & $M$ & $\mathrm{H}$ & $M$ & $\mathrm{H}$ & $M$ & $\mathrm{H}$ & $M$ & $\mathrm{H}$ & $M$ & $\mathrm{H}$ & $M$ & $\mathrm{H}$ & $M$ & $\mathrm{H}$ & M \\
\hline Segundo & 25 & 30 & 50 & 39 & 33 & 17 & 0 & 6 & 16 & 0 & 0 & 0 & 0 & 17 & 0 & 0 \\
\hline Cuarto & 0 & 23 & 67 & 53 & v & 26 & 59 & 50 & 25 & 47 & $v$ & 30 & 0 & 0 & 33 & 0 \\
\hline Sexto & 0 & 48 & 0 & 33 & 23 & 19 & 38 & 14 & 23 & 38 & 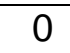 & 0 & 0 & 0 & 0 & 0 \\
\hline Octavo & 26 & 91 & 37 & 27 & 26 & 41 & 21 & 0 & 26 & 55 & 0 & 0 & 0 & 0 & 21 & 0 \\
\hline
\end{tabular}

* Para este cuadro, la "H" se refiere a Hombres y la "M" a Mujeres.

Fuente: elaboración propia de las personas autoras, 2020.

Referente al manejo y procesamiento de información utilizada para realizar trabajos académicos, se pueden ver las diferencias por nivel y semestre en los Cuadros 6 y 7 .

\section{Cuadro 6}

Diferencia en el procesador de textos y presentador de información en los niveles básico y avanzado por sexo y semestre

\begin{tabular}{|l|c|c|c|c|c|c|c|c|}
\hline \multirow{3}{*}{ Semestre } & \multicolumn{4}{|c|}{ Procesador de textos } & \multicolumn{4}{c|}{ Presentador } \\
\cline { 2 - 9 } & Nivel Básico & \multicolumn{2}{|c|}{ Nivel avanzado } & Nivel Básico & \multicolumn{2}{c|}{ Nivel avanzado } \\
\cline { 2 - 9 } & $\mathrm{H}^{*}$ & $\mathrm{M}$ & $\mathrm{H}$ & $\mathrm{M}$ & $\mathrm{H}$ & $\mathrm{M}$ & $\mathrm{H}$ & $\mathrm{M}$ \\
\cline { 2 - 9 } Segundo & 41.7 & 26.1 & 58.3 & 73.9 & 16.7 & 28.6 & 83.3 & 71.4 \\
\hline Cuarto & 25 & 16.7 & 75 & 83.3 & 8.3 & 13.3 & 91.7 & 86.7 \\
\hline Sexto & 46.2 & 14.3 & 53.8 & 85.7 & 30.8 & 14.3 & 69.2 & 85.7 \\
\hline Octavo & 21.1 & 40.9 & 78.9 & 59.1 & 10.5 & 18.2 & 89.5 & 81.8 \\
\hline
\end{tabular}

* Para este cuadro, la " $\mathrm{H}$ " se refiere a Hombres y la "M" a Mujeres.

Fuente: elaboración propia de las personas autoras, 2020. 
Bibliotecas. Vol. 40, № 1, enero - junio, 2022. EISSN: 1659-3286

URL: http://www.revistas.una.ac.cr/index.php/bibliotecas/index

\section{Cuadro 7}

Diferencia en hojas de cálculo y uso de bases de datos en niveles básico y avanzado por sexo y semestre

\begin{tabular}{|l|c|c|c|c|c|c|c|c|}
\hline \multirow{3}{*}{ Semestre } & \multicolumn{4}{|c|}{ Hoja de Cálculo } & \multicolumn{4}{c|}{ Bases de datos } \\
\cline { 2 - 9 } & \multicolumn{2}{|c|}{ Nivel Básico } & \multicolumn{2}{c|}{ Nivel avanzado } & \multicolumn{2}{c|}{ Nivel Básico } & \multicolumn{2}{c|}{ Nivel avanzado } \\
\cline { 2 - 9 } & $\mathrm{H}^{*}$ & $\mathrm{M}$ & $\mathrm{H}$ & $\mathrm{M}$ & $\mathrm{H}$ & $\mathrm{M}$ & $\mathrm{H}$ & $\mathrm{M}$ \\
\hline Segundo & 50 & 47.8 & 50 & 52.2 & 75 & 56.5 & 25 & 43.5 \\
\hline Cuarto & 50 & 36.7 & 50 & 63.3 & 33.3 & 40 & 66.7 & 60 \\
\hline Sexto & 23.1 & 19 & 76.9 & 81 & 69.2 & 61.9 & 30.8 & 38.1 \\
\hline Octavo & 36.8 & 27.3 & 63.2 & 72.7 & 36.8 & 45.5 & 63.2 & 54.5 \\
\hline
\end{tabular}

* Para este cuadro, la "H" se refiere a Hombres y la "M" a Mujeres.

Fuente: elaboración propia de las personas autoras, 2020.

Se observa que las diferencias en el manejo del procesador de texto, presentador de información y hojas de cálculo, al ser de los más utilizados para realizar actividades académicas, tanto hombres como mujeres se encuentran en los niveles avanzados. Sin embargo, en el uso de bases de datos, es en el nivel básico donde se encuentra la mayoría de la población estudiantil (con excepción de los de cuarto semestre, quienes se posicionaron en el nivel avanzado). Además, en cuanto al manejo de medios, se reportan los siguientes datos en relación con los niveles básico, intermedio y avanzado (Cuadros 8, 9 y 10):

\section{Cuadro 8}

Diferencia en manejo de Imagen por sexo y semestre

\begin{tabular}{|l|c|c|c|c|c|c|}
\hline \multirow{2}{*}{ Semestre } & \multicolumn{4}{|c|}{ Imagen } \\
\cline { 2 - 7 } & \multicolumn{2}{|c|}{ Básico } & \multicolumn{2}{c|}{ Intermedio } & \multicolumn{2}{c|}{ Avanzado } \\
\cline { 2 - 7 } & Hombre & Mujer & Hombre & Mujer & Hombre & Mujer \\
\hline Segundo & 25 & 26.1 & 58.3 & 60.9 & 16.7 & 13 \\
\hline Cuarto & 8.3 & 23.3 & 83.3 & 70 & 8.3 & 6.7 \\
\hline Sexto & 46.2 & 47.6 & 38.5 & 42.9 & 15.4 & 9.5 \\
\hline Octavo & 21.1 & 13.6 & 52.6 & 59.1 & 26.3 & 27.3 \\
\hline
\end{tabular}

* Para este cuadro, la "H" se refiere a Hombres y la "M" a Mujeres.

Fuente: elaboración propia de las personas autoras, 2020. 


\section{Cuadro 9}

Diferencia en manejo de Video por sexo y semestre

\begin{tabular}{|l|c|c|c|c|c|c|}
\hline \multirow{3}{*}{ Semestre } & \multicolumn{4}{c|c}{ Video } \\
\cline { 2 - 7 } & \multicolumn{2}{|c|}{ Básico } & \multicolumn{2}{c|}{ Intermedio } & \multicolumn{2}{c|}{ Avanzado } \\
\cline { 2 - 7 } & Hombre & Mujer & Hombre & Mujer & Hombre & Mujer \\
\hline Segundo & 27.3 & 39.1 & 45.5 & 52.2 & 27.3 & 8.7 \\
\hline Cuarto & 25 & 36.7 & 33.3 & 50 & 41.7 & 13.3 \\
\hline Sexto & 23.1 & 61.9 & 46.2 & 23.8 & 30.8 & 14.3 \\
\hline Octavo & 31.6 & 27.3 & 36.8 & 27.3 & 31.6 & 45.4 \\
\hline
\end{tabular}

Fuente: elaboración propia de las personas autoras, 2020.

\section{Cuadro 10}

Diferencia en manejo de Audio por sexo y semestre

\begin{tabular}{|l|c|c|c|c|c|c|}
\hline \multirow{3}{*}{ Semestre } & \multicolumn{5}{c|}{ Audio } \\
\cline { 2 - 7 } & \multicolumn{2}{|c|}{ Básico } & \multicolumn{2}{c|}{ Intermedio } & \multicolumn{2}{c|}{ Avanzado } \\
\cline { 2 - 7 } & Hombre & Mujer & Hombre & Mujer & Hombre & Mujer \\
\hline Segundo & 25 & 43.5 & 50 & 47.8 & 25 & 8.7 \\
\hline Cuarto & 16.7 & 33.3 & 50 & 60 & 33.3 & 6.7 \\
\hline Sexto & 15.4 & 33.3 & 61.5 & 61.9 & 23.1 & 4.8 \\
\hline Octavo & 10.5 & 22.7 & 52.6 & 45.5 & 36.8 & 31.8 \\
\hline
\end{tabular}

Fuente: elaboración propia de las personas autoras, 2020.

En general, se observó que la población estudiantil presenta un nivel ligeramente por debajo de lo esperado y que, aunque existen rubros donde hombres y mujeres se ubican en niveles iguales, existen diferencias en algunos aspectos evaluados.

\section{Discusión}

Es incuestionable que la tecnología impregnará el proceso de educación en sus diferentes agentes, subprocesos, métodos y herramientas; ya que se ha demostrado ser útil en la generación de aprendizaje significativo, mejora en la práctica docente, actualización y colaboración interinstitucional. La incorporación de las TIC en la educación es un aspecto clave 
para el desarrollo de un sistema educativo de calidad y competitivo, ya que facilita el desarrollo de las habilidades informativas y disminuye la brecha digital.

En esta investigación se encontraron datos que ofrecen un panorama sobre las habilidades tecnológicas e informativas que tiene el estudiantado de la carrera de Bibliotecología y Estudios de la Información de la UNAM; llama la atención que, aunque la mayoría tiene acceso a equipos de cómputo u otros dispositivos (INEGI 2015, 2017 y 2019), existe un 20\% de estudiantes que no lo tienen, lo cual es una cifra considerable si se toma en cuenta que es una licenciatura dedicada al manejo de la información.

Respecto a los conocimientos de procesamiento y administración de información, se reportaron niveles de habilidades altos. Es preciso mencionar que la incorporación de las TIC, desde los niveles de educación básicos, es un factor que puede estar influyendo en que la población estudiantil desarrolle las habilidades informativas desde edades tempranas, tal como lo menciona la CEPAL al referir que México es uno de los países con altos indicadores de definición e implementación de las políticas en TIC.

Como lo menciona la OECD, se deben tomar en cuenta diversos factores que ayuden en el diseño de programas educativos que incluyan el uso de las TIC; también es oportuno señalar que, en países en vías de desarrollo, como es el caso de México (señalado por la CEPAL), no basta únicamente con la asignación de recursos, además se debe priorizar que tanto estudiantes como docentes tengan las habilidades y competencias informativas requeridas.

No todos los programas de educación superior incluyen a las TIC de manera explícita en las asignaturas, existen algunos en donde se implementan de forma transversal en el currículo, dando por hecho que el cursarlas ocasionaría que los estudiantes desarrollen las habilidades informativas necesarias para responder a las necesidades y exigencias sociales y culturales, sin embargo, como lo refieren diversos autores, esto no es necesariamente una regla ni efecto directo (Salado y Álvarez, 2016; Frías, 2017; Rodríguez, Santana, Cobarrubias y Arcadia, 2018; Gómez, Alvarado, Martínez y Díaz de León, 2018; Gómez, 2019; Sánchez, 2020). 
Es necesario enfatizar que no solo se necesita realizar cambios en la infraestructura de las instituciones, sino que, además, hay que considerar el contexto social y las características personales para diseñar y establecer programas educativos que aminoren el desfase de lo que se enseña y lo que se necesita en la vida laboral.

En el caso de los participantes del estudio, se presentaron datos que indican apropiación de las TIC en más del $50 \%$ al conocer y usar recursos que les brinda su institución como el acceso a bibliotecas digitales (SIBIUNAM) y bases de datos, tal es el caso de Scielo que obtuvo el mayor puntaje en cuanto a popularidad, seguido de Library and Information Science Abstracts (LISA) e Información Bibliotecológica Latinoamericana (INFOBILA); además, se distingue una mezcla entre fuentes de recursos documentales de acceso abierto y otras del ámbito comercial, sobresaliendo que las fuentes tienden a la especialidad de la carrera;

Es interesante resaltar que alrededor del 50\% del estudiantado indica una apropiación de TIC, ya que se esperaría que por la cercanía, posesión y uso de las tecnologías se encontraran en un porcentaje más alto; sin embargo, se puede entender este fenómeno ya que no todos se encuentran en la misma etapa de uso de las tecnologías, dejando así evidenciada la brecha digital.

En términos de herramientas digitales, los estudiantes mencionaron tener conocimiento básico de aquellas que se utilizan en el aula como es el uso de correo electrónico, manejo de paquetería office, plataformas educativas y uso de redes sociales. Sin embargo, en el tema de manejo de recursos multimedia se obtuvieron niveles medios (apenas por arriba del $50 \%$ ), dejando ver de que, a pesar de que se tienen conocimientos en lo general, existen aún aspectos de las TIC que es necesario reforzar; por ejemplo, sobre los recursos digitales multimedia que circulan en la Web, un porcentaje alto de estudiantes (70\%) pronunció que dichos recursos tienen características de libre acceso o dominio público, marcando en ello un bajo nivel de conocimiento sobre los derechos reservados, o bien, sobre los permisos de uso en la red. Como bien menciona Frías (2017) al referir que no se debe dar por hecho que son jóvenes con dominio de las tecnologías y Salado y Álvarez (2016) al mencionar el uso de las TIC no asegura que el proceso educativo sea productivo. 
Debido a que el estudio se realizó en estudiantes de bibliotecología, llama la atención que una quinta parte de la población no identifique los nombres de los gestores bibliográficos siendo que deberían destacar por ser un recurso de la disciplina y que, por otra parte, el $40 \%$ evidencie no utilizarlas de forma regular en sus actividades académico-estudiantiles.

Por otro lado, se observaron diferencias importantes entre la población estudiantil en cuanto al acceso, uso, apropiación e implementación de las TIC en su proceso de educación formal, mostrando la necesidad de disminuir la brecha digital con la firme intención de impactar en su aprendizaje, es decir, pareciera que se tiene poca consciencia de cómo adquieren el conocimiento o puede ser que se esté sobrevalorando el uso de la red social en la enseñanza porque las ocupan para comunicarse, pero no como una herramienta para estudiar.

Además, para realizar un análisis profundo y sistemático de la brecha digital, como refieren algunos autores (Márquez, Acevedo y Castro, 2016, citado en Gómez, Alvarado, Martínez y Díaz de León, 2018), es necesario contemplar al personal docente quienes necesitan sensibilizarse y flexibilizar el paradigma de enseñanza tradicional al incorporar las TIC dentro y fuera del aula como un recurso viable y permanente. Además, reiterando lo que proponen Montoya, Soriano y Soriano, (2019), es necesaria la capacitación y actualización de herramientas tecnológicas para favorecer el desarrollo de habilidades informativas con uso ético y apoyar en la reducción de la brecha digital.

En particular, las estadísticas expuestas por organismos gubernamentales, internacionales o de instituciones de educación superior, sobre la cantidad de tecnologías en los hogares, o sobre los niveles de penetración del internet en la sociedad, continuarán siendo, sin duda alguna, indicadores cuantitativos importantes a considerar en la toma de decisiones para intentar disminuir o abatir la brecha digital.

\section{Conclusiones y recomendaciones}

El presente estudio aporta información acerca de la brecha digital en estudiantes universitarios del área de humanidades, situación que es relevante ante la poca literatura encontrada en ese tipo de población. Los participantes se pueden considerar como nativos digitales ya que su 
generación ha estado en contacto con las TIC desde edades tempranas, como tener dispositivos fijos y móviles con conexión a internet que facilitan el acceso a la información de forma permanente, sin embargo, se presentaron datos que muestran que aunque saben la importancia de proteger sus dispositivos y cuentas, poseen poca información de ello y por lo tanto, realizan pocas acciones relacionadas con la seguridad de sus dispositivos, situación que ha facilitado la presencia de robo de identidad en diferentes plataformas.

Por otro lado, se obtuvieron datos que muestran que los estudiantes tienen los recursos necesarios para estar conectados, sin embargo, se desconoce si estos son óptimos y cubren las necesidades académicas que se exige en la formación universitaria.

En relación con el uso de bibliotecas digitales, los resultados mostraron que la mitad utiliza los recursos informativos disponibles en el SIBIUNAM, tanto de aquellos que se encuentran en las colecciones generales como en las especializadas. Llama la atención que un 10\% no utiliza los recursos digitales que la institución académica ofrece.

Si bien los jóvenes en la actualidad se encuentran inmersos en contextos tecnológicos y el uso de las redes sociales es un aspecto común y de dominio como forma de comunicación, llama la atención que, aunque la mayoría de los jóvenes utiliza las redes sociales, solo un pequeño porcentaje las usa como fuente de información académica y motor de búsqueda.

En términos generales, los estudiantes de Bibliotecología de la UNAM reportaron niveles medios de conocimientos de procesamiento y administración de información, niveles medios en conocimientos de recursos multimedia y en cuanto a habilidades informativas se reportó que se encuentran en proceso de incorporarse y consolidarse en su repertorio como agentes activos en su proceso de aprendizaje. Este dato es relevante ya que enfatiza la necesidad de desarrollar intervenciones para los estudiantes universitarios porque, si bien los participantes utilizaban la tecnología para cuestiones educativas, se esperaba que tuvieran niveles más altos en su uso y en habilidades informacionales debido a que la mayoría tenía acceso tanto a dispositivos como a internet, además que desde hace algunas generaciones, la educación básica le ha dado importancia al uso de la tecnología. 
El estudio presenta algunas limitaciones, por ejemplo, se reportan datos de una sola evaluación, por lo que para futuras investigaciones sería importante realizar estudios longitudinales para conocer la evolución de las habilidades durante la licenciatura; otra limitación que tuvo el estudio fue el tamaño de la muestra, debido a que es pequeña no representa a toda la población de la carrera de Bibliotecología.

Existe poca literatura que muestre datos específicos de un área de conocimiento, por lo que los datos presentados abren líneas de investigación que pueden contribuir al diseño, implementación y evaluación de intervenciones que favorezcan el desarrollo de las habilidades informativas tomando en cuenta el sexo y etapa de avance en la licenciatura.

\section{Referencias}

American Library Association (1988). Marco de referencia para habilidades para el manejo de la información en la educación superior. Recuperado de http://www.ala.org/searchresults?as q=habilidad\%20informativa

Azamar, A. (2016). La integración de la tecnología al Sistema Educativo Mexicano: sin plan ni rumbo. Reencuentro: análisis de problemas universitarios. 72, 11-25.

Byerly, G. And Brodie, C. (1999). Information Literacy Skills Models: Defining The Choices. In Learning And Libraries In An Information Age: Principles And Practice. Ed. Barbara K. Stripling, Englewood: Littleton: Libraries Unlimited, pp. 54-82.

Campbell, S. (2004). "Defining Information Literacy in the 21st century". En IFLA 70th Conference. Recuperado de http://www.ifla.org/IV/ifla70/papers/059e-Campbell.pdf

Castellanos Domínguez, O. F., Claudia Nelcy Jiménez Hernández y Karen Patricia Domínguez Martínez. (2009). Competencias tecnológicas: bases conceptuales para el desarrollo tecnológico en Colombia. Revista Ingeniería e Investigación. 29(1), 133-139.

Chartered Institute of Library and Information Professionals (2004). Alfabetización en información: la definición de CILIP (UK). Boletín de la Asociación Andaluza de Bibliotecarios. (77) 79-84.

Durán, R.E., Rivero, L.F., Alfaro, B., y Gayosso, S. (2018). Tecnología educativa en México 2013-2016: análisis de la política pública y aproximaciones a la realidad nacional. En A.J., 
Argüelles, C.M., Vicario, y P. Gómez. (comps). Política y gestión de tecnología educativa en México. pp. 13-33. México: Instituto Politécnico Nacional.

Freire, J.; Mora, L (2009). Competencias informacionales e informáticas en el ámbito universitario. [Presentación]. VII Jornadas CRAl, 4-5 de junio.

Frías, L. (2017). La universidad impulsa la alfabetización tecnológica, programa de formación permanente. Gaceta UNAM. 4821, 3.

Gobierno de México (s/f). Dirección de tecnologías de información. Recuperado de https://www.gob.mx/ceav/acciones-y-programas/direccion-de-tecnologias-de-lainformacion

Gómez, D. C. (2019). Una aproximación a la evolución de la brecha digital entre la población joven en España (2006-2015). Revista Española de Sociología. 28(1).

Gómez, D. A., Alvarado, R. A., Martínez, M., y Díaz de León, C. (2018). La brecha digital: una revisión conceptual y aportaciones metodológicas para su estudio en México. Entreciencias: diálogos en la Sociedad del Conocimiento. 6(16), http://dx.doi.org/10.22201/enesl.20078064e.2018.16.62611

González, N. (2010). Competencias del bibliotecario 2.0. Bibliotecarios 2.0: experimento y aprendizaje. Recuperado de http://bibliotecarios2-0.blogspot.com/2009/04/competenciasdel-bibliotecario-20.html

Hernández, R.M. (2017). Impacto de las TIC en la educación: Retos y perspectivas. Propósitos y Representaciones. 5(1), 325-347. Recuperado de https://revistas.usil.edu.pe/index.php/pyr/article/view/149

Hinostroza, J. E. y Labbé, C. (2011). Políticas y prácticas de informática educativa en América Latina $y$ el Caribe. Santiago de Chile: Naciones Unidas. https://repositorio.cepal.org/bitstream/handle/11362/6182/S2011014 es.pdf?sequence=1\& isAllowed $=y$

Instituto Nacional de Estadística y Geografía (INEGI). (2015). Encuesta Intercensal 2015. Recuperado de https://www.inegi.org.mx/programas/intercensal/2015/

Instituto Nacional de Estadística y Geografía (INEGI). (2017). Encuesta sobre la Percepción Pública de la Ciencia y la Tecnología 2017. Recuperado de https://www.inegi.org.mx/programas/enpecyt/2017/ 
Instituto Nacional de Estadística y Geografía (INEGI). (2019). Estadísticas a propósito del día mundial del Internet. Recuperado de

https://www.inegi.org.mx/contenidos/saladeprensa/aproposito/2019/internet2019 Nal.pdf

Lau, J. (2007). Directrices sobre desarrollo de habilidades informativas para el aprendizaje permanente. Boca del Río, Veracruz, México: IFLA. Recuperado de: https://www.ifla.org/files/assets/information-literacy/publications/ifla-guidelines-es.pdf

Ministerio de Educación - ENLACES (2013). Matriz de habilidades TIC para el aprendizaje. Recuperado de

https://bibliotecadigital.mineduc.cl/bitstream/handle/20.500.12365/2165/mono-

95x.pdf? sequence $=1$ \&isAllowed $=y$

Montoya Chávez, L. M., Soriano Fonseca, J., y Soriano Fonseca, J. (2019). Brecha digital: alfabetización digital docente en las instituciones de educación superior (IES). Revista Internacional de Aprendizaje en la Educación Superior,6(1), 9-20. Recuperado de https://journals.eagora.org/revEDUSUP/article/view/2000

Olivares Carmona, K. M., Angulo Armenta, J., Torres Gastelú, C. A., Madrid García, E. M. (2016). Las TIC en educación: metaanálisis sobre investigación y líneas emergentes en México. Apertura (Guadalajara, Jal.), 8(2), 100-115.

Organización para la Cooperación y el Desarrollo Económicos (OCDE) (2002). La definición y selección de competencias clave. Resumen ejecutivo. Recuperado de https://www.deseco.ch/bfs/deseco/en/index/03/02.parsys.78532.downloadList.94248 .DownloadFile.tmp/2005.dscexecutivesummary.sp.pdf

Owusu-Ansah, E. K. (2003). Information literacy and the academic library: a critical look at a concept and the controversies surrounding it. The Journal of Academic Librarianship, 29(4), 219-230.

Prensky, M. (2001). Digital native, digital immigrants: digital native immigrants. On the horizon, 9(5). Recuperado de http://www.marcprensky.com/writing/Prensky\%20\%20Digital\%20Natives,\%20Digital\%20Immigrants\%20-\%20Part1.pdf

Rodríguez, H., Santana, J.D., Cobarrubias, N., y Arcadia, J.R. (2018). El uso de las TIC y los estudiantes universitarios. Revista Digital de Tecnologías Informáticas y Sistemas, 2(2). Recuperado de https://www.redtis.org/index.php/Redtis/article/view/15 
Salado Rodríguez, L., y Álvarez Flores, E. P. (2016). Uno de los rostros de la brecha digital en la universidad: las prácticas institucionalizadas en el uso de las TIC. Revista Internacional de Educación $y$ Aprendizaje, 4(1). Recuperado de https://journals.eagora.org/revEDU/article/view/555/134

Sánchez Jiménez, A. (2020, febrero 22). Ingresan a la UNAM sólo 10 de cada 100; van al concurso $130 \quad$ mil. La Jornada. Recuperado de https://www.jornada.com.mx/ultimas/sociedad/2020/02/22/ingresan-a-la-unam-solo-10-decada-100-van-al-concurso-130-mil-6684.html

Secretaría de Comunicaciones y Transportes. (2001). Sistema Nacional E-México. Recuperado de https://www.gob.mx/cms/uploads/attachment/file/67638/CAP-07.pdf

Secretaría de Comunicaciones y Transportes. (2012). Acciones para el fortalecimiento de la banda ancha y las tecnologías de la información y comunicación. [México]: Gobierno de México. http://www.sct.gob.mx/uploads/media/AFBAyTICs.pdf

Secretaría de Educación Pública (s/fa). Programa de Desarrollo eEucativo 1995 - 2000 Educación Media Superior y Superior. Recuperado de http://publicaciones.anuies.mx/pdfs/revista/Revista97_S3A2ES.pdf

Secretaría de Educación Pública (s/fb). Libro Blanco Programa Enciclomedia 2006-2012. Recuperado de https://www.sep.gob.mx/work/models/sep1/Resource/2959/4/images/LB\%20Enciclomedia. pdf

Secretaría de Educación Pública (s/fc). Agenda digital educativa. Recuperado de https://infosen.senado.gob.mx/sgsp/gaceta/64/2/2020-02-05-

1/assets/documentos/Agenda_Digital_Educacion.pdf.

Secretaría de Educación Pública (1995). Programa de Desarrollo Educativo 1995-2000. Revista Latinoamericana de Estudios Educativos. 25(3), 133-147.

Secretaría de Educación Pública (2007). Programa Sectorial de Educación 2007-2012. México: Secretaría de Educación Pública.

Webber, S. (2006). Information Literacy in Higher Education. En: Stopar, K. and Rabzeljl. (Eds) Informacijska Pismenost med teorijo in prakso: vloga visokošolskih in specialnih knjižnic: Zbornik prispevkov. [Information Literacy between theory and practice: the role of academic 
Bibliotecas. Vol. 40, № 1, enero - junio, 2022. EISSN: 1659-3286

URL: http://www.revistas.una.ac.cr/index.php/bibliotecas/index

and special libraries: Proceedings.] Ljubljana: ZBDS. Recuperado de https://www.academia.edu/1003839/Information literacy in higher education

\section{Notas de las personas autoras}

El artículo es resultado parcial del Proyecto PAPIIT Programa de Alfabetización Informacional para estudiantes de la Facultad de Filosofía y Letras, UNAM (IA402119).

Selene Violeta Castillo Rojas, Colegio de Bibliotecología, UNAM, México; correo electrónico: selenecastillo@filos.unam.mx, ORCID 0000-0002-8333-7383

Carlos Omar Sánchez Xicotencatl, Facultad de Psicología, UNAM, México; correo electrónico: omarxicotencatl@comunidad.unam.mx; ORCID 0000-0002-7767-0040

Alberto Castro Thompson, Instituto de Investigaciones Bibliográficas, UNAM; correo electrónico: acastro@unam.mx; ORCID 0000-0001-7298-2742

Lina Escalona Ríos, Facultad de Filosofía y Letras, UNAM, México; correo electrónico: linae@unam.mx, ORCID 0000-0002-5109-4612 